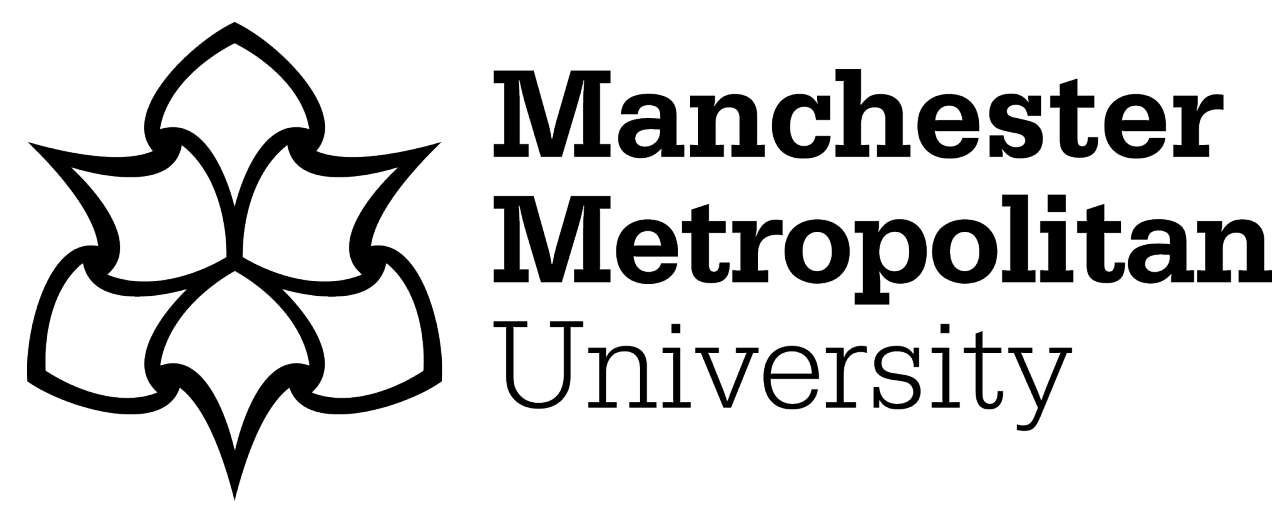

Randviir, Edward P ORCID logoORCID: https://orcid.org/0000-0001-72528494 and Banks, Craig E ORCID logoORCID: https://orcid.org/0000-00020756-9764 (2015) Electrode substrate innovation for electrochemical detection in microchip electrophoresis. Electrophoresis, 36 (16). pp. 1845-1853. ISSN 0173-0835

Downloaded from: https://e-space.mmu.ac.uk/596005/

Version: Accepted Version

Publisher: Wiley

DOI: https://doi.org/10.1002/elps.201500153

Please cite the published version 


\title{
Electrode Substrate Innovation for Electrochemical Detection in Microchip Electrophoresis
}

\author{
Edward P. Randviir and Craig E. Banks* \\ Faculty of Science and Engineering, School of Chemistry and the Environment, \\ Division of Chemistry and Environmental Science, Manchester Metropolitan University, \\ Chester Street, Manchester M1 5GD, Lancs, UK
}

Submission to: Electrophoresis

*To whom correspondence should be addressed.

Email: c.banks@mmu.ac.uk; Tel: ++(0)1612471196; Fax: ++(0)1612476831

Website: www.craigbanksresearch.com

Received: 22-Mar-2015; Revised: 11-May-2015; Accepted: 11-May-2015

This article has been accepted for publication and undergone full peer review but has not been through the copyediting, typesetting, pagination and proofreading process, which may lead to differences between this version and the Version of Record. Please cite this article as doi: 10.1002/elps.201500153.

This article is protected by copyright. All rights reserved. 


\section{Abstract}

Microchip Electrophoresis (ME) represents the next generation of miniaturised electrophoretic devices and carry benefits such as significant improvement in analysis times, lower consumption of reagents and samples, flexibility, and procedural simplicity. The devices provide a separation method for complex sample matrices and an on-board detection method for the analytical determination of a target compound. The detection part of ME is increasingly leaning towards electrochemical methods, thus the selectivity and sensitivity of detection in ME is dependent upon the chosen working electrode composition in addition to operating conditions of the chip such as separation voltage. Given the current plethora of electrode materials that are available, there exists a possibility to creatively integrate electrodes into ME. This review will overview the application of several electrode materials, from the old through to the new. A particular recent focus has been the selectivity element of MEs overcome with the use of enzymes, carbon composites, and screen-printed technologies.

\section{Introduction}

One problem with the real world application of analytical techniques lies within the sample matrix that target analytes arrive within. For example, clinical samples are contained within blood, urine, or saliva, while environmental samples could be anything from mud, water, air, rock, sludge, or sands. The complex nature of such matrices leads to a high possibility of interference or noise from a detection system, thus a host of analytical procedures are utilised to process a sample, such as acid digestion, liquid or solid phase extractions, or even electrophoretic separation, prior to the actual measurement step in order to isolate the intended chemical species one wishes to observe. This multi-phase protocol can be time consuming and requires samples to be transported to a lab, when ideally the injection, separation, and measurement would be all done at the point of sample collection.

The aim of Microchip Electrophoresis (ME) is to scale specific lab techniques (such as Capillary Electrophoresis, CE) into one portable handheld unit with a centimetre-sized geometry, therefore integrating multiple sample handling processes a combine with some measurement steps [1]. The separation part of ME was focussed on in detail prior to 2005, with the technology maturing quite rapidly [1]. However scale-down detection strategies for such technologies unfortunately lagged behind. Many chips still required the use of LaserInduced Fluorescence (LIF) or Mass Spectrometry (MS) for the detection of target species, both of which require large off-chip measurement systems. Ideally one would combine both 
the separation and detection elements into ME, therefore the external use of LIF or MS is contradictory to this philosophy. Research has since been directed towards the development of a detection strategy that can be fabricated into a chip, thus completing the design of a selfcontained microsystem when combined with electrophoretic separation and injection. Electrochemistry has thus attracted considerable attention as a chemical detection method in ME devices $[2,3]$ due to the possibility of on-chip integration using electrodeposited or printed circuitry, in addition to its relative inexpensiveness, speed of measurement, and portability benefits when compared to LIF or MS.

The purpose of this opening review is to assess electrode substrates that are commonly implemented for the purpose of electrochemical detection in ME devices and demonstrate how researchers have developed new methods to incorporate electrodes into microchips. The review is designed to focus primarily upon potentiostatic types of electrochemical detection modes using controlled potential techniques such as those often implemented for electroanalytical applications. The review discusses specific examples of electrode substrates applied for the detection or target analytes, thus the reader requires a good understanding of electrochemical redox reactions for complete understanding of the work discussed within. 


\section{Microchip Electrophoresis}

In order to explain how an ME chip can function, we make reference to a chip created for the detection of creatinine; a Schematic is given in Figure 1 [4]. The device utilises CE and conductivity measurements to separate and detect creatinine within a sample of human serum. Serum samples are inserted at point 1 and subject to a high voltage injection step, allowing the matrix to migrate into the device. The potential for injection is applied using electrodes at points $\mathrm{A}$ and $\mathrm{B}$. The charged species migrate down channel 3 towards the separation channel in the chip at point 4 . Electrodes C and D apply a secondary electric field that allows separation of the target analyte. It is at this point that the target species is physically removed/isolated from the sample matrix, which in theory allows ease of detection. The electrodes at point 6 detect the separated chemical species, in this case using conductivity. The conductivity of the sample is plotted as a function of time in an electropherogram. In this approach the retention time of the target species in required to be known, unless of course the working electrode is tailored to only transduce responses for specified targets. As in all electrochemical measurements, a conductivity measurement would be arbitrary without some reference value for the conductivity measurement to be compared against. Therefore, point $\mathrm{E}$ allows for conductivity measurements of the sample without prior separation. The conductivity of the separated analyte is thus standardised against the conductivity of the bulk solution in this case. If we assume that ME devices generally operate using similar protocols (high voltage injection followed by CE separation), we can focus specifically upon the detection aspect of the chips, as depicted by point 6 in Figure 1. The detection and reference electrodes must be tailored specifically for different applications and has been the focus of much work since the birth of ME devices, particularly in recent times. The remainder of the review focusses upon a short selection of integrated electrodes utilised for ME devices and strategies employed to design, produce, and improve working electrodes for ME purposes. 


\section{Working Electrodes for Microchip Electrophoresis}

Many ME systems utilise working electrodes engineered for a specific target analyte. CE electrodes, such as platinum, copper, or silver chloride electrodes are designed to separate matrices by their $m / z$ ratios [5], but this review focusses upon working electrodes that are designed to detect the target species under scrutiny. A working electrode in this review is defined as an electrode that is responsible for measuring the concentration of an intended target species. Potentiostatic detection electrodes in ME devices generally employ amperometric systems, since there is a wide acceptance that such methods are acceptable for detecting analytes contained in liquid-based effluents [1]. Yet, voltammetric measurements are equally possible. This section discusses some of the first electrodes implemented for microfluidic electrochemical detection purposes, and proceeds to discuss the progression of electrode development from the 1990s to the present day.

\section{Early Devices}

The first reports of ME working electrodes date back to 1996 by Gavin and Ewing [6]. Their photolithographic platinum electrodes were embedded into a chip constructed from quartz. An amperometric method was demonstrated to detect dopamine using channel flow electrophoresis with electrochemical detection; something that was previously problematic due to the electric fields imposed in capillary electrophoresis affecting electrochemical results. The chip was designed in a manner that allowed for less electric field interference from injection and separation, by etching the electrodes in a recess some $25 \mu \mathrm{m}$ from the height of the channel flow. This way the electric field in the capillary is less likely to interfere with the dynamic electrochemistry that takes place at the electrode surfaces. However their works infers that one should exercise caution that the recess height is not too deep. This is because the mass transport will completely change and target analytes can be trapped within the recesses, allowing for atypical electrochemical responses consisting of convoluted Faradaic and non-Faradaic contributions. Though their pioneering work was designed to prove that electrochemistry and CE could be combined into one unit, they also used an array of 100 electrodes that provided some temporal information about the chemical system, in terms of monitoring changes in environment with respect to time, particularly in biological cases. The internal heights and electrode separations were the focus of later work by the same authors as they continued their quest to use amperometric platinum electrodes to detect 
dopamine using the same technology [7]. Their investigations proved that the platinum arrays displayed a non-uniform sensitivity when detecting dopamine. This phenomenon is depicted in Figure 2B, where it can be seen that a more concentrated injection of dopamine into the chip results in a longer current depletion time than for a less concentrated injection. It is clear that the two cases exhibit different sensitivities, thus complicated analysis would have to be performed if the sample concentration was unknown. Furthermore, results were only valid after a normalisation step that requires a pre-treatment stage prior to analysis, meaning that though the concept is valid, it comes with some major practical limitations that require addressing.

There were further reports about the integration of microelectrodes into microchannels for in-stream electrochemical detection of target analytes shortly thereafter. Darling et al. developed a microchip from silica containing etched channels via a wet chemical process using EDP (a mixture of ethylene diamine and pyrocatechol) [8], reporting on-board electrochemical detection via anodic striping voltammetry. Their work demonstrated again that dopamine derivatives could be separated and detected using different electrochemical methods on-board of a microchip. Work by Woolley et al. also utilised similar chips to separate and detect neurotransmitters and DNA [9]. A pitfall to their work was the separation voltage significantly interfered with the results and therefore relatively small voltages had to be applied across the chip, meaning analysis times were longer than desired. Yet this was proven to be offset by changing the working and reference electrode spacing; the closer the detection electrodes were together, the less pronounced the interference phenomenon. This is intuitive because the potentials in the detection area will have less time to be influenced by the separation voltages. The gold working electrodes were used in a single-electrode system, unlike previous cases that desired to create an electropherogram typical of concentration or mass monitoring. The advantage to this method is a simpler analysis procedure, but lacks the robustness of the array method because it requires prior calibration and knowledge of analyte retention times under the specific conditions monitored. Other electrodes were investigated by Wang and co-workers, such as sputtered gold electrodes [10] and screen-printed carbon electrodes that were externally mounted upon microchips [11]. The latter exhibited better sensitivities than previously investigated on-board strategies. Later works furthered electrochemical detection in microchips such as the report by Martin and co-workers regarding dual-electrode electrochemical detection using Poly(DiMethylSiloxane), PDMS, fabricated microchips [12]. Their work was the first report of a dual-electrode microchip system, and also the first report 
of electrochemical detection using microchips fabricated from PDMS. At the time this was an inherent advantage because chips could be fabricated from a master template repeatedly and much quicker than glass chips that were etched using hydrofluoric acid. The dual-electrode strategy allows for individual tailoring of working electrode surfaces to detect differing components of a mixture, something that is near impossible on a single working electrode. This is particularly useful if two compounds have similar $m / z$ ratios, because they will reach the detector at different times.

Unfortunately prior to 2005 there were very few reports of electrochemical detection in $\mathrm{ME}$ and this was probably a limitation to the overall development of ME because many detection methods such as LIF and MS are not portable, even if they exhibit enhanced sensitivity and chemical reliability than a standard electrochemical method. However, the early attempts opened up clear research pathways towards providing electrochemical detectors. Some researchers opted for glass substrates that exhibit durability but lack the speed of preparation that a PDMS substrate offers. Photolithography was generally employed to apply platinum electrodes into such devices, yet gold electrodes were also suitable for some applications, while sputtered and printed electrodes were also useful as disposable options to be inserted into a chip, rather than built into the chip. The biggest problems were with the separation voltage that is required for CE; a technical solution was offered by Gavin and Ewing but is not used by others in this era. The potential for growth and development in the field therefore hinges on chip design, electric field interference, electrode separation, single/dual mode electrodes or arrays, fundamental understanding of diffusion regimes, and finally the selectivity of the working electrodes, as it is clear that currents will be produced for many biological species that undergo oxidation at noble metal surfaces under the influence of electric potential. The field responded to these hurdles and many reports have emerged since 2005 that have enhanced electrochemical detection in microchip technology. The remainder of the review will focus upon more recent electrode materials for $\mathrm{ME}$ applications.

\section{Modern Devices}

The boom in ME technology triggered a surge in academic literature regarding electrochemical ME post-2005. Several reviews prior to this focussed upon electrochemical detection, such as the contribution by Lacher et al., but though the work was novel then, the electrodes described normally lacked the selectivity required for application in the field [13]. In particular, there was a focus upon standard electrode substrates such as gold, platinum, and 
glassy carbon, but with the evolution of electrochemistry, such standard substrates are seen much less due to a lack of selectivity. Wang also provided reviews in 2002 and 2005, both of which had a similar theme to the present review, but with a more self-fulfilling reference list $[1,14]$. There was a shift towards more selective ME devices utilising several electrode substrates thereafter. A flurry of reviews focussing upon several defined aspects of electrochemical ME was observed, such as Trojanowicz's review of flow analysis strategies [15], while others turned attention towards aptamers [16], and others take a general detection direction [17]. This section encompasses electrode substrates and the engineering of electrochemical microchips for specific applications, with a particular focus on dynamic current-potential techniques such as voltammetry and amperometry.

\section{Enzymatic Electrodes}

A simplified philosophy of ME is to scale and laboratory techniques, combining the injection, separation, and detection parts of an analytical method into one handheld unit. Thus it is no surprise that the potential applications span forensic science, clinical chemistry, and environmental science as these areas require on-the-spot analysis methods from complex matrices. The first example of ME electrochemistry concerns the latter field. The monitoring of phenolic compounds is a significant importance environmentally because they are harmful to environmental systems and human health, yet many agricultural and petrochemicals still contain phenols. Mayorga-Martinez et al. therefore designed a microfluidic chip with an electrochemical detection port that selectively detects phenols [18]. Their screen-printed carbon electrode design incorporates the use of tyrosinase, an enzyme that selectively reacts with phenolic compounds, on board a composite designed to transduce enzymatic processes into current transients. The benefits of the device depicted in Figure 3 is that the electrode can be repeatedly replaced between measurements, meaning that cost effective screen-printed electrodes offer a unique, cheap, and portable application for ME electrochemical detection. The disposable screen-printed sensor, which transduces electrochemical reactions into currents, simply has to be inserted into the device (Figure 3B) prior to use, and connected to a potentiostat. Such disposable sensors cost the user a matter of pence. The modern potentiostat is a handheld battery-powered device that can be transported to wherever required. In such an application, the microchip and potentiostat can potentially be combined and transferred to the site of an environmental sample, reducing sample contamination. A combination of CE and electrochemistry is required for such samples in order to separate the analyte from the matrix first so that electrochemical interference from background analytes is reduced. 
Application of ME to the clinical industry is just as important as to the environment. One example is that of the hydrogen peroxide sensor designed by Matharu and co-workers [19], which is cleverly utilised to understand how specific cell cultures interact with alcohol. The ME detection system, depicted in Figure 4, is fabricated in a multi-step system that requires several components in order to successfully transduce chemical interaction events into current signals. A gold electrode is modified with silane, polyethylene glycol, and HorseRadish Peroxidase (HRP). The saline surrounding the electrode in Figure 4 serves as a bed for hepatocytes, which are liver cells responsible for detoxification of blood. When alcohol is injected into the device, the hepatocytes respond by breaking the alcohol down into hydrogen peroxide and acetal, in a 2 electron 2 proton process that takes place at the cell membrane surfaces. The liberated extracellular hydrogen peroxide is broken down by the HRP fixed on the gold electrode surface and transduced into a current signal. The array of modified electrodes utilised allows for a time-dependent electropherogram that could be used to study the rate of alcohol degradation, and also the introduction of antioxidants is studied to observe the effect of antioxidants on peroxide formation and oxidative stress levels.

Within the last three years there have been many examples of enzyme-based electrochemical microfluidic devices that are worth mentioning. Instead of discussing each application individually, Table 1 has been provided for interested readers to view different enzymatic detection strategies for a range of applications including cholesterol, HIV, and glucose [20-24].

\section{Graphene-Based Electrodes}

In line with modern electrochemistry, graphene-based electrodes have also been exhaustively investigated for application in ME devices. One such report was by Chua and Pumera, who focussed upon reduced graphene oxides for use as detection electrodes for dopamine and catechol [25]. These electrodes are created by suspending lab-synthesized graphenes in an organic solvent, which is drop-casted upon a glassy carbon electrode using a standard Eppendorf pipette. The reduced graphenes (thermally, chemically, and electrochemically reduced) displayed no inherent advantage over normal carbon electrodes such as glassy carbon for the detection of dopamine and catechol in such systems. The authors attribute this to an increase in background current exhibited by the electrode composites, yet it is likely that the increased basal nature of graphene compared to other graphitic materials is responsible for poor peak currents. Furthermore, the electrode is essentially being utilised as a channel flow electrode in such cases, so the constant laminar 
flow will have a detrimental impact upon the drop-casted surface by mechanically stripping the composite from the surface, due to no covalent linkage of the graphene to the substrate.

Solution-based graphenes are also utilised to detect cholesterol by Ruecha and coworkers [26]. Their method uses the conducting properties of graphene to improve the conductivity of a polymer electrode. The graphenes were mixed with polyvinylpyrrolidone and polyaniline, creating graphene-infused nanostructures that can be electrosprayed upon a substrate. In their work, the mixture is electrosprayed upon paper with cholesterol oxidase, thus cholesterol is broken down, liberating hydrogen peroxide as a biproduct that is detected amperometrically, similar to many other cases. The utility of graphene in this example is to make a conducting polymer that can be sprayed easily onto a ME device, thus creating sensitive and selective electrodes that are easy to mould onto the desired substrate.

Carbohydrates are also useful to detect and often appear in complicated matrices. Thus, work by Zhang et al. focussed upon fabricating graphene and nickel nanoparticles that could be grafted upon resin microspheres for the detection of specific carbohydrates in $\mathrm{ME}$ systems [27]. Their work exhibited a sensitivity towards glucose, sucrose, and fructose of 200-300 nA mM $\mathrm{m}^{-1}$ using an amperometric detection method with a pipette tip as an electrode case. Further examples of graphene-based microfluidic working electrodes are given in Table $2[28-32]$.

\section{Carbon Nanotube-Based Electrodes}

Drop-casting of carbon nanomaterials upon substrates is by no means limited to graphenes. In fact, Carbon NanoTubes (CNTs) were fabricated and studied at length long before graphenes ever were and are still researched today. CNTs such as Single-Walled and Multi-Walled CNTs (MWCNT/SWCNT) are generally utilised by researchers to reduce activation potentials for electrochemical processes, thus making electrodes more efficient, stable, and longer lasting. CNTs allow this because their anisotropic structures orient themselves in a manner in which the electronically concentrated zones are exposed as the electrode surface [33]. This is the opposite phenomenon to graphene, where the structures orient themselves so that the electronically sparse basal planes are exposed as the electrode surface. The advantageous electrochemistry of CNTs has therefore attracted much attention for electrodes in ME devices.

One such example of an integrated CNT electrode on a microchip is by $\mathrm{Xu}$ and coworkers, who incorporate CNTs into a polystyrene matrix in order to fabricate a conducting polymer electrode, which can be applied for the detection of active ingredients in herbal 
medicines [34]. The polymer is mounted upon a copper wire and cased within a silica tube to define a working electrode with a finite diameter of $2 \mathrm{~mm}$. The fabricated electrode was inserted into a chip designed to accommodate an electrode of such a diameter. The CNT composite provided a reduced activation potential for rutin of $+0.49 \mathrm{~V}$, compared to $+0.67 \mathrm{~V}$ when compared to a graphite composite of the same design. This reduction in peak potential is a result of the electronic anisotropy as mentioned previously, and allows a lower energy activation in the chip that is advantageous in terms of peak resolution. These in-situ approaches are commonplace for microchip electrode design. Other works report SWCNT electrodes obtained by press-transfer technology on PMMA substrates for ME applications [35]. Through careful optimisation of the conditions, the press-transferred electrodes can exhibit excellent repeatability, an extreme resistance to fouling (unlike in the case of graphenes), remarkable signal-to-noise characteristics, and a well-defined linear concentration dependence. Other works using CNTs are provided in Table 3 [36-41].

\section{Screen-Printed Electrodes}

Modern research focusses upon many substrates for detection of target species, yet sometimes the simplest technologies are the most useful. Screen-printed carbon electrodes, sometimes referred to as thick-film electrodes [1], exhibit a host of advantages over thin-film electrodes and conventional electrodes such as glassy carbon and gold. They are cheap, mass producible, disposable electrodes that often require no recourse or pre-treatment steps. They are far easier to fabricate than thin-film electrodes, are more reliable in terms of mechanical stress, and require simple and relatively inexpensive old printing technologies instead of deposition techniques such as physical vapour deposition or chemical vapour deposition. It is for these reasons that screen-printed technologies find uses in many areas including clinical [42, 43], forensic [44-46], and environmental science [47].

In terms of microchips, there have already been examples described above that utilise screen-printed electrodes in microchip detectors. One such example is that of the Prussianblue modified screen-printed electrode that is utilised to detect glucose, as reported by Sekar and co-workers [48]. A paper-based substrate was utilised in this example as an ideal matrix to house glucose oxidase, providing the specificity for glucose. The Prussian blue is incorporated due to its ability to reduce hydrogen peroxide at a lower potential, thus allowing the sensor to be operated using low energies that will not disturb other chemical constituents of a sample matrix. 
Of course, it would be better to create a detection system that was free from enzymes because the electrode would not be limited by the strict operating conditions that are required to maximise enzymatic interactions. Unfortunately most examples are restricted to enzymeon-paper coupled with enzymeless electrochemical detection. Yang and co-workers employ a novel screen-printed system for microfluidic usage using such a system, where platinum nanoparticles act as the sensor for hydrogen peroxide on a screen-printed electrode [49]. A specific sensitivity of $10 \mu \mathrm{A} \mathrm{mM} \mathrm{mm}^{-1} \mathrm{~cm}^{-2}$ indicates a very useful sensing platform for hydrogen peroxide and may be the superior choice of electrode for any case that uses enzymatic processes as the majority create hydrogen peroxide as its primary biproduct. However this strategy may not be suitable for devices wishing to exhibit more than a few uses, because hydrogen peroxide is known to foul substrates quite corrosively. In terms of a disposable one shot sensor, these strategies are amenable, however.

Laser printing is also a viable option, as demonstrated in work by Coltro et al [50]. Such devices used laser printed electrodes for the detection part of the ME device, a strategy that is quite simple in principle. Their device was demonstrated to detect iodide and ascorbate to levels as low as 500 and $1800 \mathrm{nmol} \mathrm{L}^{-1}$, respectively. There also exist other approaches, such as the use of copper nanowires as electrode materials for the ME sensing of galactosemia [51], or a similar approach for the determination of saccharides in honey [52], highlighting the seemingly endless possibilities that electrode design can offer for $\mathrm{ME}$ applications. Microchip electrophoresis-copper nanowires for fast and reliable

\section{determination of monossacharides in honey samples}

There are simply too many examples of electrodes to surmise in one review alone. This review as tried to capture some of the electrodes that can be incorporated directly into ME, yet other reviews have found many more avenues to explore. For example, gold nanoparticles are utilised by some researchers in DNA applications [53]. A review by Pumera summarises how ME can be utilised for the detection of explosives [54], while Matysik's review focusses upon the advances of amperometry within ME applications [55]. Dungchai's review also looks at electrochemical detection strategies, this time in paper-based devices [3]. Finally, Xu et al focus their review upon different electrochemical detection modes utilised within ME [56]. 


\section{Concluding Remarks}

A range of electrochemical detection strategies are available that are feasible on the micro-scale and have been successfully applied to ME. From old electrodes such as gold and platinum, these cases supply the advantage of good sensitivities, but with the compromise of a complicated production procedure such as lithography and they come at a good deal of expense. Thus other strategies such as, enzymes, printed carbons, and graphenes were focussed upon by the field in order to reduce electrode size, cost, improve reproducibility and be mass produced, while maintaining a level of selectivity for specified applications. Many electrodes succeed in this endeavour and create strategies that may be suitable for such applications, but others less so. Solution-based graphenes are unreliable due to weak noncovalent linkages to electrode substrates allowing mechanical stripping under high voltage laminar flow. Enzymatic technologies are selective but require specific conditions and storage that may limit their practical application in the long term. Screen-printed electrodes hold an advantage of being mass-producible but require specific tailoring of surfaces in order to detect molecules selectively. As seen within this short review, researchers tend to opt for a combination of different routes to tailor the electrode response, most of which utilise the hydrogen peroxide pathway and a chronoamperometric detection method. There are questions over the long term suitability of this given that hydrogen peroxide forms radicals and attacks many different substrates, but a continuation of this philosophy is likely because such electrodes give the highest selectivity in a given application. The improvement of electrochemical detection methods will continue and will likely form the first choice method for ME detection in the coming years. 


\section{References}

[1] Wang, J., Electroanalysis 2005, 17, 1133-1140.

[2] Zimmerman, W. B., Chemical Engineering Science 2011, 66, 1412-1425.

[3] Dungchai, W., Chailapakul, O., Henry, C. S., Analytical Chemistry 2009, 81, 5821-5826.

[4] Ávila Muñoz, M., Eijkel, J., Floris, A., Staal, S., Ríos, Á., Berg, A. v. d., 15th International Conference on Miniaturized Systems for Chemistry and Life Sciences, Chemical and Biological Microsystems Society, Seattle, Washington 2011, pp. 1206-1208.

[5] Neihof, R. E. X., Schuldiner, S., Nature 1960, 185, 526-527.

[6] Gavin, P. F., Ewing, A. G., Journal of the American Chemical Society 1996, 118, 89328936.

[7] Gavin, P. F., Ewing, A. G., Analytical Chemistry 1997, 69, 3838-3845.

[8] Darling, R., Yager, P., Weigl, B., Kriebel, J., Mayes, K., in: Harrison, D. J., van den Berg,

A. (Eds.), Micro Total Analysis Systems '98, Springer Netherlands 1998, pp. 105-108.

[9] Woolley, A. T., Lao, K., Glazer, A. N., Mathies, R. A., Analytical Chemistry 1998, 70, 684-688.

[10] Wang, J., Tian, B., Sahlin, E., Analytical Chemistry 1999, 71, 3901-3904.

[11] Wang, J., Tian, B., Sahlin, E., Analytical Chemistry 1999, 71, 5436-5440.

[12] Martin, R. S., Gawron, A. J., Lunte, S. M., Henry, C. S., Analytical Chemistry 2000, 72, 3196-3202.

[13] Lacher, N. A., Garrison, K. E., Martin, R. S., Lunte, S. M., ELECTROPHORESIS 2001, 22, 2526-2536.

[14] Wang, J., Talanta 2002, 56, 223-231.

[15] Trojanowicz, M., Analytica Chimica Acta 2009, 653, 36-58.

[16] Xu, Y., Yang, X., Wang, E., Analytica Chimica Acta 2010, 683, 12-20.

[17] Baker, C. A., Duong, C. T., Grimley, A., Roper, M. G., Bioanalysis 2009, 1, 967-975.

[18] Mayorga-Martinez, C. C., Hlavata, L., Miserere, S., López-Marzo, A., Labuda, J., Pons, J., Merkoçi, A., ELECTROPHORESIS 2013, 34, 2011-2016.

[19] Matharu, Z., Enomoto, J., Revzin, A., Analytical Chemistry 2013, 85, 932-939.

[20] Ali, M. A., Srivastava, S., Solanki, P. R., Reddy, V., Agrawal, V. V., Kim, C., John, R., Malhotra, B. D., Sci. Rep. 2013, 3.

[21] Parsajoo, C., Kauffmann, J.-M., Talanta 2013, 109, 116-120.

[22] Shafiee, H., Jahangir, M., Inci, F., Wang, S., Willenbrecht, R. B. M., Giguel, F. F., Tsibris, A. M. N., Kuritzkes, D. R., Demirci, U., Small 2013, 9, 2553-2563.

[23] Malon, R. S. P., Chua, K. Y., Wicaksono, D. H. B., Corcoles, E. P., Analyst 2014, 139, 3009-3016.

[24] Gu, S., Lu, Y., Ding, Y., Li, L., Song, H., Wang, J., Wu, Q., Biosensors and Bioelectronics 2014, 55, 106-112.

[25] Chua, C. K., Pumera, M., Electroanalysis 2013, 25, 945-950.

[26] Ruecha, N., Rangkupan, R., Rodthongkum, N., Chailapakul, O., Biosensors and Bioelectronics 2014, 52, 13-19.

[27] Zhang, R., Lu, S., Zhang, L., Chen, G., Journal of Chromatography A 2014, 1374, 261267.

[28] Kwon, O. S., Lee, S. H., Park, S. J., An, J. H., Song, H. S., Kim, T., Oh, J. H., Bae, J., Yoon, H., Park, T. H., Jang, J., Advanced Materials 2013, 25, 4177-4185.

[29] Kumar, R., Jahan, K., Nagarale, R. K., Sharma, A., ACS Applied Materials \& Interfaces 2015, 7, 593-601.

[30] Wu, Y., Xue, P., Hui, K. M., Kang, Y., Biosensors and Bioelectronics 2014, 52, 180187. 
[31] Lee, S. H., in: Wang, Y. Z. (Ed.), Quantum, Nano, Micro Technologies and Applied Researches, Applied Mechanics, Singapore 2013, pp. 102-107.

[32] Wang, Y., Ge, L., Wang, P., Yan, M., Ge, S., Li, N., Yu, J., Huang, J., Lab on a Chip 2013, 13, 3945-3955.

[33] Banks, C. E., Davies, T. J., Wildgoose, G. G., Compton, R. G., Chemical Communications 2005, 829-841.

[34] Xu, J., Zhang, H., Chen, G., Talanta 2007, 73, 932-937.

[35] Vilela, D., Garoz, J., Colina, Á., González, M. C., Escarpa, A., Analytical Chemistry 2012, 84, 10838-10844.

[36] Yao, X., Wu, H., Wang, J., Qu, S., Chen, G., Chemistry - A European Journal 2007, 13, 846-853.

[37] Vilela, D., Martin, A., Gonzalez, M. C., Escarpa, A., Analyst 2014, 139, 2342-2347.

[38] Wisitsoraat, A., Sritongkham, P., Karuwan, C., Phokharatkul, D., Maturos, T., Tuantranont, A., Biosensors and Bioelectronics 2010, 26, 1514-1520.

[39] Kang, X., Mai, Z., Zou, X., Cai, P., Mo, J., Analytical Biochemistry 2007, 363, 143-150.

[40] Pumera, M., Merkoçi, A., Alegret, S., ELECTROPHORESIS 2007, 28, 1274-1280.

[41] Li, X., Chen, Z., Zhong, Y., Yang, F., Pan, J., Liang, Y., Analytica Chimica Acta 2012, 710, 118-124.

[42] Randviir, E. P., Banks, C. E., Sensors and Actuators B: Chemical 2013, 183, 239-252.

[43] Randviir, E. P., Kampouris, D. K., Banks, C. E., Analyst 2013, 138, 6565-6572.

[44] Smith, J. P., Metters, J. P., Kampouris, D. K., Lledo-Fernandez, C., Sutcliffe, O. B., Banks, C. E., Analyst 2013, 138, 6185-6191.

[45] Smith, J. P., Metters, J. P., Irving, C., Sutcliffe, O. B., Banks, C. E., Analyst 2014, 139, 389-400.

[46] Smith, J. P., Metters, J. P., Khreit, O. I. G., Sutcliffe, O. B., Banks, C. E., Analytical Chemistry 2014, 86, 9985-9992.

[47] Figueiredo-Filho, L. C. S., Janegitz, B. C., Fatibelilo-Filho, O., Marcolino-Junior, L. H., Banks, C. E., Analytical Methods 2013, 5, 202-207.

[48] Chandra Sekar, N., Mousavi Shaegh, S. A., Ng, S. H., Ge, L., Tan, S. N., Sensors and Actuators B: Chemical 2014, 204, 414-420.

[49] Yang, J., Nam, Y.-G., Lee, S.-K., Kim, C.-S., Koo, Y.-M., Chang, W.-J., Gunasekaran, S., Sensors and Actuators B: Chemical 2014, 203, 44-53.

[50] Tomazelli Coltro, W. K., Fracassi da Silva, J. A., Torres da Silva, H. D., Richter, E. M., Furlan, R., Angnes, L., do Lago, C. L., Mazo, L. H., Carrilho, E., ELECTROPHORESIS 2004, 25, 3832-3839.

[51] García, M., Alonso-Fernández, J. R., Escarpa, A., Analytical Chemistry 2013, 85, 91169125.

[52] García, M., Escarpa, A., ELECTROPHORESIS 2014, 35, 425-432.

[53] Shiddiky, M. J. A., Shim, Y.-B., Analytical Chemistry 2007, 79, 3724-3733.

[54] Pumera, M., ELECTROPHORESIS 2006, 27, 244-256.

[55] Matysik, F.-M., Microchim Acta 2008, 160, 1-14.

[56] Xu, J.-J., Wang, A.-J., Chen, H.-Y., TrAC Trends in Analytical Chemistry 2007, 26, 125132. 
Figure 1 Schematic diagram of the microfluidic creatinine device in Reference [4]. The different components are as follows: (1) sample opening with applied sample droplet; (2) evaporation reservoir; (3) injection channel for injection of cations by moving boundary electrophoresis; (4) double-T injector; (5) reservoir with gas bubble for liquid expansion control; (6) conductivity detection electrodes; (A and B) high-voltage injection anode and cathode; (C and D) high-voltage separation anode and cathode; (E) electrodes for the determination of the sample conductivity.

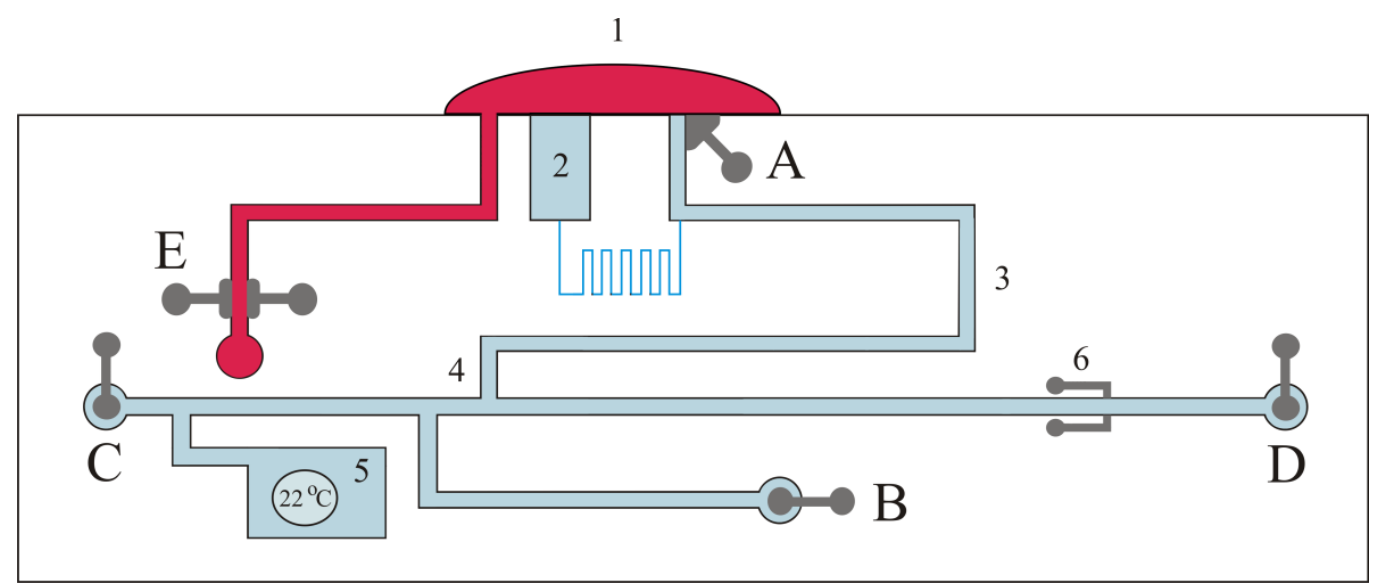


Figure 2 Different analyte concentrations monitored with the microfabricated electrophoresis-electrochemical array detection scheme. (A) Plugs of dopamine (0.665 or $3.46 \mathrm{mM} ; 45 \mathrm{~s}$ duration) were injected from a sample vial into a fused silica capillary $(10 \mu \mathrm{m}$ internal diameter, $70 \mathrm{~cm}$ length) that was coupled to a buffer-filled rectangular channel $(8 \mu \mathrm{m}$ internal height, $4.8 \mathrm{~cm}$ length). The capillary and channel were filled with MES buffer (25 $\mathrm{mM}, \mathrm{pH}$ 5.9) prior to the start of the experiment. The capillary and channel voltages used were $28 \mathrm{kV}$ and $1500 \mathrm{~V}$, respectively. The capillary was moved at a rate of $0.2 \mathrm{~s} / \mathrm{step}$. The separation current was $0.19 \mathrm{~mA}$. Detection was at $+0.8 \mathrm{~V}$ vs. $\mathrm{Ag} / \mathrm{AgCl}$. (B) Threedimensional plot of the electropherogram shown in (A). The three-dimensional plot displays changes in current depletion with respect to concentration of the injected analyte, where the spikes on the left represent the higher concentration (slower current depletion) and the spikes on the right represent the lower concentration (faster current depletion). Reprinted from Reference [7] with permission from the American Chemical Society.

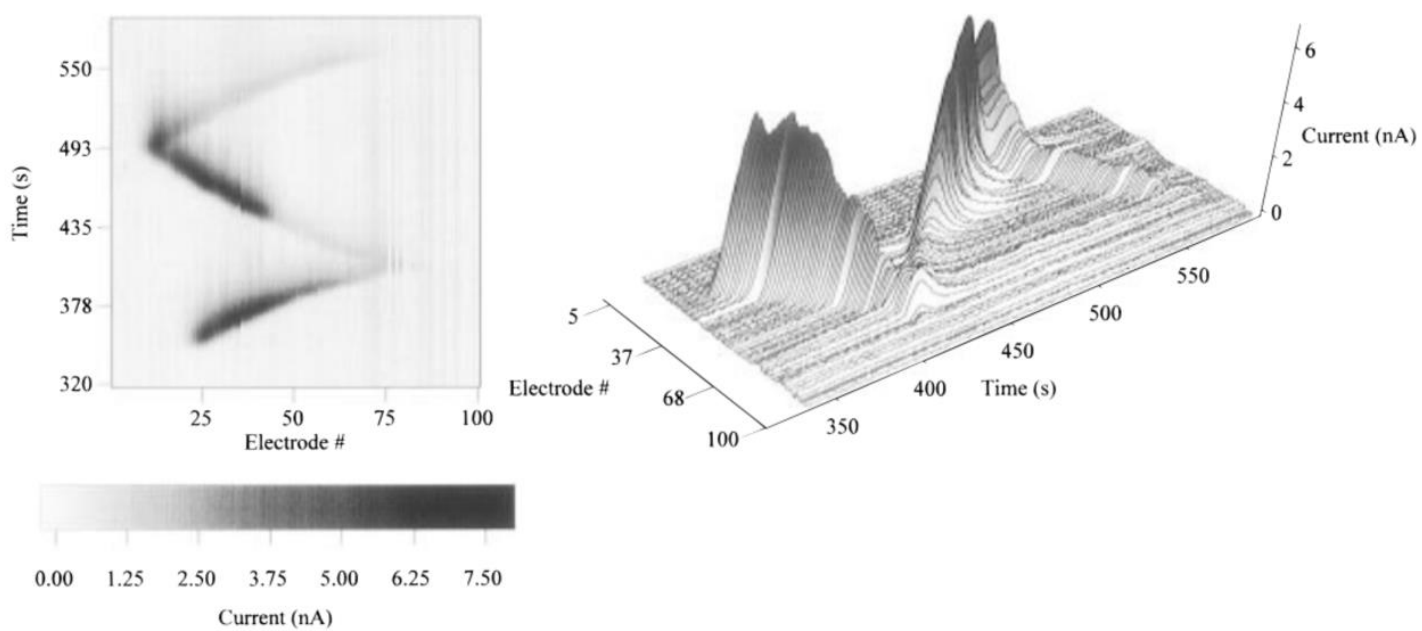


Figure 4 Steps 1-3. Fabrication of enzymatic microstructures on gold electrodes. Steps 5-6. Culture and injury of hepatocytes followed by electrochemical detection of extracellular peroxide. Reprinted from Reference [19] with permission from the American Chemical Society.

$\underline{\text { Step } 1}$

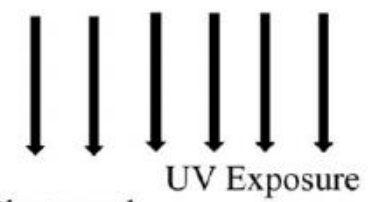

Photomask

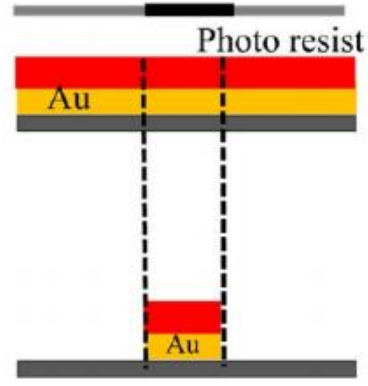

$\underline{\text { Step } 2}$

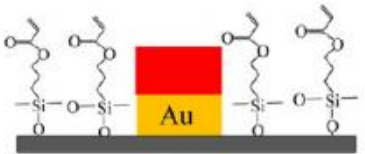

Silane modification
Step 3

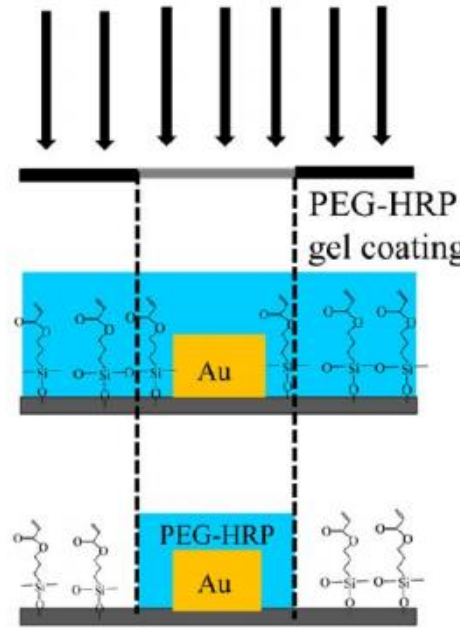

Step 4

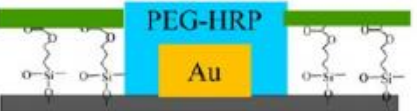

$\underline{\text { Step } 5}$
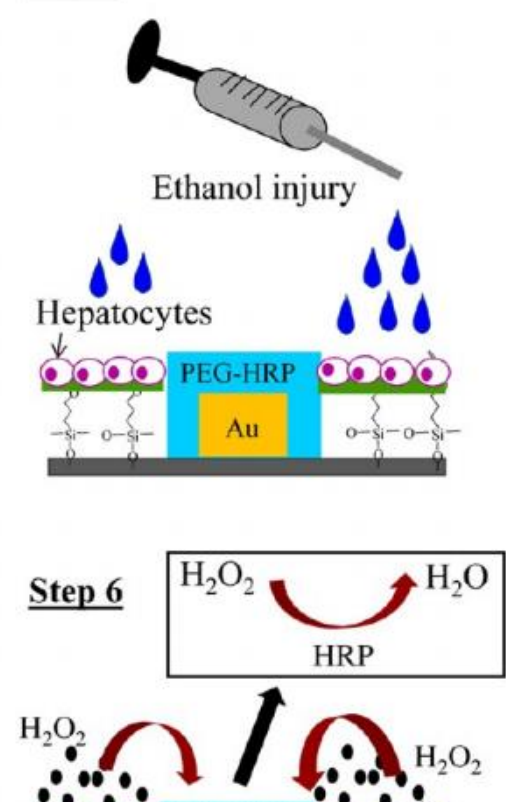
Oer PEG-HRP 0010 
Table 1 Electrochemical microchip technologies employing enzymatic detection methods.

\begin{tabular}{llrr}
\hline Electrode & Application & Sensitivity & Comments \\
\hline $\begin{array}{l}\text { 1. Multi-walled carbon } \\
\text { nanotubes }\end{array}$ & Cholesterol & $2.2 \mathrm{~mA} \mathrm{mM}^{-1} \mathrm{~cm}^{-2}$ & Use of covalent linkages \\
2. Nickel oxide nanoparticles & & $\begin{array}{r}\text { between terminating species on } \\
\text { nanotubes connect enzyme to } \\
\text { 3. Cholestrol oxidase and }\end{array}$ & the substrate \\
cholesterol esterase & & Selective amino acid \\
1. Acetylcholinesterase upon & Thiocholine & $0.597 \mu \mathrm{A} \mathrm{mM}^{-1}$ & determination \\
gold & & &
\end{tabular}

2. Silver

1. Gold

HIV

N/A Impedimetric sensor relies upon

2. Magnetic beads non-Faradaic interactions,

3. HIV antibodies producing current transients similar to antibody/antigen interactions

1. Screen-printed carbon

Lactate

$0.317 \mu \mathrm{A} \mathrm{mM}^{-1}$

Amperometric measurement electrode

2. Lactate oxidase detects hydrogen peroxide produced from the reaction of lactate oxidase with lactate from saliva on a cotton-based substrate

1. Platinum

Glucose ca. $0.25 \mu \mathrm{A} \mathrm{mM}^{-1}$

Platinum black improves surface

2. Platinum black area for signal transduction

3. Glucose oxidase 
Table 2 Electrochemical microchip technologies employing graphene-based sensing electrodes.

\begin{tabular}{|c|c|c|c|}
\hline Graphene & Application & Comments & Ref. \\
\hline $\begin{array}{l}\text { Single-layer micropatterned } \\
\text { graphene nanohybrids }\end{array}$ & HIV antibodies & $\begin{array}{r}\text { Current response indicative of HIV } \\
\text { antibodies }\end{array}$ & [28] \\
\hline $\begin{array}{l}\text { Polyaniline-wrapped } \\
\text { graphene }\end{array}$ & Electro-osmotic pump & $\begin{array}{l}\text { Non-sensing application but worthy } \\
\text { of a mention due to its innovation }\end{array}$ & [29] \\
\hline Reduced graphene oxide & Cancer biomarkers & $\begin{array}{l}\text { Four cancner biomarker sensing } \\
\text { routes developed using the same } \\
\text { paper-based methodology }\end{array}$ & [30] \\
\hline Graphene and gold & Mercury & $\begin{array}{r}10 \text { ppm detection limit for } \\
\text { environmental sensing }\end{array}$ & [31] \\
\hline $\begin{array}{l}\text { Graphene-modified gold } \\
\text { paper electrode }\end{array}$ & DNA hybridization & Detect DNA in femtomolar levels & {$[32]$} \\
\hline
\end{tabular}


Table 3 Electrochemical microchip technologies employing CNT-based sensing electrodes.

CNT

Press-transferred SWCNT

CNTs for in-channel flow

enzyme immobilization

SWCNT/MWCNT

Copper nanocluster and

SWCNT electrode

MWCNT and cobalt

hexacyanoferrate

\section{Application}

250 s response time with high accuracy

Low cross sensitivity towards interfering

Dopamine

Glucose

Hydrazine
Comments

species

Ref.

Qualitative study displays the potential electrocatalytic properties of CNTs

Linear range spans four orders of

$$
\text { magnitude }
$$

Useful for environmental monitoring 